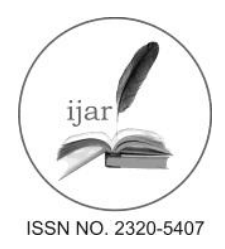

Journal homepage: http://www.journalijar.com

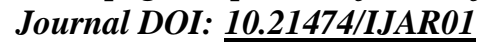

INTERNATIONAL JOURNAL

OF ADVANCED RESEARCH

RESEARCH ARTICLE

\title{
CHANGES OF SERUM CARDIAC MARKERS AFTER ACUTE ATTACK OF MYOCARDIAL INFARCTION
}

DR.Charmi Vyas*(M.D.pathology,tutor.),DR.Shweta B.Upadhyay**(M.D.pathology,associate professor).

$\begin{array}{ll}\text { Manuscript Info } & \text { Abstract } \\ \text { Manuscript History. } & \end{array}$

Received: 17 February 2016

Final Accepted: 29 March 2016

Published Online: April 2016

Key words:

*Corresponding Author

DR.charmi vyas.

Copy Right, IJAR, 2016,. All rights reserved.

\section{Introduction:-}

Acute myocardial infarction (AMI) is one of the most common diagnosis in hospitalized patients in industrialized countries. The early (30 days) mortality rate from AMI is approximately 30 percent, with more than half of these deaths occurring before individual reaches the hospital ${ }^{1}$. So it has become one of the major causes of mortality in the world at present, and it is for this reason various researches are being done for its diagnosis and prognostic assessment. Serum enzyme estimation is an important tool for it $^{2}$.

In recent years, the incidence of MI has increased in the population because of change in lifestyle, urbanization, increase in mental stress, inadequate physical exercise and diet rich in lipid. It has also been observed that there is sudden increase in acute MI in young subjects mostly in second and third decades of life who show increased morbidity and mortality. Hence, this will become a dreadful condition for the next millennium. ${ }^{2}$

AMI accounts to cause about $1 / 3^{\text {rd }}$ of total deaths in united states. So it has become very much necessary for us to diagnose AMI as quickly as possible because earlier diagnosis of attack of MI has great impact on treatment and its outcome. The use to 3 diagnostic methods to aid in clinical diagnosis of AMI, which includes electrocardiogram, enzyme analysis, non-invasive techniques and others which could help to provide early diagnosis of AMI. ${ }^{3}$

Accuracy of ECG diagnosis of AMI is not more than 80 percent and it is often found that in cases of early AMI the ECG could be normal. So the enzyme estimation has become of immense utility in early diagnosis.

Rapid appearance of CK-MB in the serum after AMI and improves specificity for myocardial injury provided by measurement of MB isoenzyme quickly established in the clinical setting ${ }^{4}$.

Troponin (cardiac troponin I and $\mathrm{T}$ ) are very sensitive and specific indicators of damage to heart muscle (myocardium). They are measured in the blood to differentiate between unstable angina and myocardial infarction in patients with chest pain or acute coronary syndrome. A patient who had suffered from AMI would have an area of damaged heart muscle and so would have elevated cardiac troponin level in blood ${ }^{5}$. 
In our study, we collected samples of 100 patients admitted for complain of chest pain to measure Troponin I, CK$\mathrm{MB}$, hsCRP, LDH and to see changes in these cardiac biomarkers with primary end point of sensitivity and specificity of these cardiac biomarkers.

Aims and objective:-

1. To study changes in serum cardiac markers after acute attack of MI.

2. To determine the sensitivity and specificity of Troponin I, CK-MB, hsCRP, LDH.

3. To establish usefulness of enzymes in early diagnosis of AMI.

\section{Material and methods:-}

Patients are selected from those attending department of medicine, G.G. Hospital, M.P. Shah Medical College, Jamnagar. Blood samples of patients were taken to measure the serum LDH, Troponin I, CRP, CK-MB levels by ELISA (enzyme linked immunosorbent assay) and their levels are compare with clinical condition, ECG changes and prognosis of the patients.

\section{Observation and results:-}

Demographic profile of the patients

Table 1:

\begin{tabular}{|c|c|c|c|}
\hline Age group (yrs) & Num of Male patients & Num of Female patients & Total number of patients \\
\hline $\mathbf{2 1 - 3 0}$ & 02 & 02 & 04 \\
\hline $\mathbf{3 1 - 4 0}$ & 06 & 00 & 06 \\
\hline $\mathbf{4 1 - 5 0}$ & 14 & 02 & 16 \\
\hline $\mathbf{5 1 - 6 0}$ & 28 & 10 & 38 \\
\hline $\mathbf{6 1 - 7 0}$ & 14 & 04 & 18 \\
\hline $\mathbf{7 1 - 8 0}$ & 09 & 04 & 13 \\
\hline $\mathbf{8 1 - 9 0}$ & 03 & 02 & 05 \\
\hline Total & $\mathbf{7 6}$ & $\mathbf{2 4}$ & $\mathbf{1 0 0}$ \\
\hline
\end{tabular}

Incidence of risk factors

Table 2:

\begin{tabular}{|l|l|l|l|l|l|l|}
\hline \multirow{2}{*}{ Risk factor } & Num of positive cases & \multicolumn{4}{l|}{ Num of negative cases } \\
\cline { 2 - 7 } & Male & Female & Total & Male & Female & Total \\
\hline Smoking & 58 & 0 & 58 & 18 & 24 & 42 \\
\hline Hypertension & 54 & 20 & 74 & 22 & 4 & 26 \\
\hline IHD & 50 & 22 & 72 & 26 & 2 & 28 \\
\hline Diabetes mellitus & 32 & 23 & 55 & 44 & 1 & 45 \\
\hline
\end{tabular}

Table 3:

\begin{tabular}{|c|c|c|c|}
\hline Condition & Male & Female & Total \\
\hline Acute MI & 36 & 19 & 25 \\
\hline Unstable angina & 19 & 06 & 10 \\
\hline LRTI & 06 & 04 & 06 \\
\hline COPD & 06 & 00 & 04 \\
\hline GERD & 03 & 01 & 25 \\
\hline
\end{tabular}

Total 100 patients having chest pain were studied out of which 55 were diagnosed as having acute attack of MI and were treated accordingly. Conditions associated with chest pain

Table 4:

\begin{tabular}{|c|c|c|c|}
\hline $\begin{array}{c}\text { Serum cardiac } \\
\text { markers done }\end{array}$ & Num of positive cases & Num of negative cases & Total no. Of cases \\
\hline Trop-i & 55 & 00 & 55 \\
\hline CK-MB & 49 & 06 & 55 \\
\hline Hs-CRP & 40 & 15 & 55 \\
\hline LDH & 36 & 19 & 55 \\
\hline
\end{tabular}


Table 5:

\begin{tabular}{|l|c|c|c|c|}
\hline & Troponin I & CK-MB & P value & Significance \\
\hline Sensitivity & $100 \%$ & $89.1 \%$ & 0.002 & HS \\
\hline Specificity & $100 \%$ & $88.8 \%$ & 0.0017 & HS \\
\hline
\end{tabular}

NS - Non significant, S - significant, HS - Highly significant

When applying chi-square $\left\{\mathrm{X}_{2}\right\}$ test to compare sensitivity and specificity of Troponin I and CK-MB the difference was statistically highly significant. ( $\mathrm{P}$ value $<0.05)$.

Table 6:

\begin{tabular}{|l|c|c|c|c|}
\hline & Troponin I & hs-CRP & P value & Significance \\
\hline Sensitivity & $100 \%$ & $72.7 \%$ & $<0.0001$ & HS \\
\hline Specificity & $100 \%$ & $62.2 \%$ & $<0.0001$ & HS \\
\hline
\end{tabular}

NS - Non significant, S - significant, HS - Highly significant

When applying chi-square $\left\{\mathrm{X}_{2}\right\}$ test to compare sensitivity and specificity of Troponin I and hs-CRP the difference was statistically highly significant. $(\mathrm{P}$ value $<0.0001)$.

Table 7:

\begin{tabular}{|l|c|c|c|c|}
\hline & Troponin I & LDH & P value & Significance \\
\hline Sensitivity & $100 \%$ & $65.4 \%$ & $<0.0001$ & HS \\
\hline Specificity & $100 \%$ & $60 \%$ & $<0.0001$ & HS \\
\hline
\end{tabular}

- $\quad$ NS - Non significant, S - significant, HS - Highly significant

When applying chi-square $\left\{\mathrm{X}_{2}\right\}$ test to compare sensitivity and specificity of Troponin I and hs-CRP the difference was statistically highly significant. (P value $<0.0001)$.

\section{Discussion:-}

\section{Changes in Troponin I in patients complaining of chest pain:-}

In prsent study, it was found that out of 55 patients who actually diagnosed to have acute myocardial infarction, Troponin I was elevated in all 55 patients. While in 45 patients who diagnosed to have other condition than acute myocardial infarction, Troponin I was elevated in none of them. (table 4).

In present study, sensitivity and specificity of Troponin I for diagnosis of acute myocardial infarction was $100 \%$.

\section{These results are comparable to other studies:}

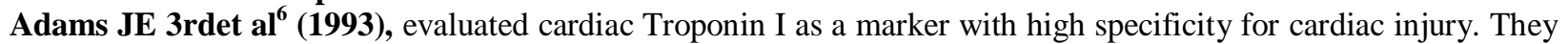
concluded that elevations of cTnI are highly specific for myocardial injury. Use of cTnI should facilitate distinguishing whether elevations of MBCK are due to myocardial or skeletal muscle injury.

Adams JE $3^{\text {rd }}$ et $\mathbf{~ a l}^{7}(\mathbf{1 9 9 4})$, done a study on comparable detection of acute myocardial infarction by creatine kinase MB isoenzyme and cardiac troponin I. They concluded that cTnI and CK-MB had statistically indistinguishable diagnostic accuracies for the detection of acute myocardial infarction.

Brogan GX Jret $\mathbf{a l}^{\mathbf{8}}$ (1997), done a study on evaluation of a new assay for cardiac Troponin I vs Creatine kinaseMB for the diagnosis of acute myocardial infarction. They concluded cTn-I was as sensitive and specific for AMI as was CK-MB in ED patients who presented within 24 hours of symptom onset. However, cTn-I was more sensitive in patients who presented $>$ or $=24$ hours after symptom onset. Elevations of either marker within 6 hours of symptom onset predict an increased risk of complications and/or need for interventions.

Shazib Pervaiz et $\mathbf{a l}^{\mathbf{9}}$ (1997), done a comparative analysis of cardiac Troponin I and Creatine Kinase-MB as markers of acute myocardial infarction. They concluded that cTnI is an excellent marker for detecting and ruling out AMI, because it has better specificity and a wider diagnostic window than the accepted standard, CK-MB. 
Durdi Qujeq ${ }^{10}$ (1999), done a study on rapid diagnosis of acute myocardial infarction. Consecutive 150 patients admitted to the coronary care unit was studied. They concluded that measurement of CTn-I accurately detects MI in patients and should facilitate the diagnosis and management of such patients.

Ross G et al ${ }^{11}$ (2000), done a study on Troponin I-sensitivity and specificity for the diagnosis of acute myocardial infarction. They concluded that when a troponin I level greater than $0.6 \mathrm{ng} / \mathrm{mL}$ was used as a positive value, compared to CK-MB and ECG using either time zero or time 6 hours, the sensitivity was $94 \%$ and specificity was $81 \%$. When troponin I greater than $2.0 \mathrm{ng} / \mathrm{mL}$ was used to define a positive test, the sensitivity was $85 \%$ and specificity was $91 \%$ when compared to CK-MB and ECG.

Kiran R. Bagaleet $\mathbf{a l}^{\mathbf{1 2}}$ (2014), done a study on Role of CK-MB and Troponin-I in Diagnosing Non-ST-Elevation Myocardial Infarction. They concluded that Troponin-I can identify the minimal cardiac damage which will be useful for the physician to start immediate intervention.

Table 8:studies on sensitivity and specificity of troponin I

\begin{tabular}{|l|c|c|c|}
\hline Authors & Year & Sensitivity & Specificity \\
\hline${\text { Adams JE } 3^{\text {rd }} \text { et al }^{84}}^{\text {Apple FS et al }}{ }^{85}$ & 1994 & $96.6 \%$ & $94.9 \%$ \\
\hline Jaffe AS et al $^{87}$ & 1995 & $100 \%$ & $91.9 \%$ \\
\hline Shazib Pervaiz et al & 1996 & $90 \%$ & --- \\
\hline Ross G et al $^{94}$ & 1997 & $95 \%$ & $97.4 \%$ \\
\hline Mahalaxmi S. Petimani et al $^{98}$ & 2000 & $85 \%$ & $91 \%$ \\
\hline In present study $^{98}$ & 2013 & $96 \%$ & $98 \%$ \\
\hline
\end{tabular}

These data suggest that Troponin $I$ is very sensitive and specific cardiac biomarker in diagnosis of acute myocardial infarction.

Changes in CK-MB in patients complaining of chest pain:-

In present study it was found that out of 55 patients who actually diagnosed to have acute myocardial infarction, CK-MB was elevated in all 49 patients. In 6 patients in spite of having acute myocardial infarction it remained in normal range. While in 45 patients who diagnosed to have other condition than acute myocardial infarction, CKMB was elevated in 5 patients and in 40 patients it remained in normal range. (table 4 ).

In present study sensitivity and specificity of CK-MB for diagnosis of acute myocardial infarction was $89.1 \%$ and $88.8 \%$ respectively. When applying chi-square $\left\{\mathrm{X}_{2}\right\}$ test to compare sensitivity and specificity of CK-MB with Troponin I the difference was statistically highly significant. (P value $<0.05$, table 7 ).

These results are comparable to other studies:

Adams JE $3^{\text {rd }}$ et $^{\mathbf{a l}^{7}}$ (1994), done a study on comparable detection of acute myocardial infarction by creatine kinase MB isoenzyme and cardiac troponin I. They concluded that cTnI and MBCK had statistically indistinguishable diagnostic accuracies for the detection of acute myocardial infarction.

Brogan GX Jr et $\mathbf{a l}^{\mathbf{8}}$ (1997), done a study on evaluation of a new assay for cardiac Troponin I vs Creatine kinaseMB for the diagnosis of acute myocardial infarction. Patients who had elevations in either CK-MB or cTn-I within 6 hours of symptom onset were at increased risk for cardiovascular complications and/or interventions (CK-MB, OR 5.8; cTn-I, OR 6.3). So they concluded cTn-I was as sensitive and specific for AMI as was CK-MB in ED patients who presented within 24 hours of symptom onset. However, cTn-I was more sensitive in patients who presented > or $=24$ hours after symptom onset. Elevations of either marker within 6 hours of symptom onset predict an increased risk of complications and/or need for interventions.

Shazib Pervaiz et al $^{\mathbf{9}}$ (1997), done a comparative analysis of cardiac Troponin I and Creatine Kinase-MB as markers of acute myocardial infarction. They concluded that $\mathrm{cTnI}$ is an excellent marker for detecting and ruling out AMI, because it has better specificity and a wider diagnostic window than the accepted standard, CK-MB. 
Janice Zimmerman et $\mathbf{a l}^{\mathbf{1 3}}(\mathbf{1 9 9 9})$, done a diagnostic marker cooperative study for the diagnosis of myocardial infarction. They found that with each marker as the diagnostic standard, CK-MB subforms and myoglobin remained the most sensitive for early diagnosis. So they concluded that the CK-MB subform assay alone or in combination with a troponin reliably triages patients with chest pain and should lead to improved therapy and reduced cost.

Bock JL et al ${ }^{14}(\mathbf{1 9 9 9})$, done an evaluation of CK-MB isoform analysis for early diagnosis of myocardial infarction. They concluded that analysis of CK-MB by high-voltage electrophoresis is an effective method for rapid diagnosis of MI, with the isoform analysis enhancing early sensitivity.

Table 9: studies on sensitivity and specificity of ck-mb.

\begin{tabular}{|l|c|c|c|}
\hline Authors & Year & Sensitivity & Specificity \\
\hline Apple FS et al $^{85}$ & 1995 & $100 \%$ & $85.6 \%$ \\
\hline Fitzgerald RL et al $^{86}$ & 1996 & $81 \%$ & $97 \%$ \\
\hline Shazib Pervaiz et al $^{90}$ & 1997 & $96.4 \%$ & $85.8 \%$ \\
\hline Jenice Zimmerman et al $^{91}$ & 1999 & $91 \%$ & $89 \%$ \\
\hline Bock JL et al $^{92}$ & 1999 & $92 \%$ & $92 \%$ \\
\hline In present study $^{9}$ & --- & $89.1 \%$ & $88.8 \%$ \\
\hline
\end{tabular}

These data suggest that CK-MB is very sensitive cardiac biomarker for diagnosis of acute myocardial infarction but its sensitivity and specificity are not as much as Troponin I. As CK-MB reaches its normal value within 2-3 days it is helpful in diagnosis of reinfarction.

Changes in hs-CRP in patients complaining of chest pain:-

In present study, it was found that out of 55 patients who actually diagnosed to have acute myocardial infarction, hsCRP was elevated in all 40 patients. In 15 patients in spite of having acute myocardial infarction it remained in normal range. While in 45 patients who diagnosed to have other condition than acute myocardial infarction, hs-CRP was elevated in 17 patients and in 28 patients it remained in normal range. (table 4).

In present study sensitivity and specificity of hs-CRP for diagnosis of acute myocardial infarction was $72.7 \%$ and $62.2 \%$ respectively. When applying chi-square $\left\{\mathrm{X}_{2}\right\}$ test to compare sensitivity and specificity of hs-CRP with Troponin I the difference was statistically highly significant. (P value $<0.0001$, table 9 ).

These results are comparable to other studies:

Zohair Al Aseriet $\mathbf{a l}^{15}$ (2014), done a study on relationship of high sensitivity C-reactive protein with cardiac biomarkers in patients presenting with acute coronary syndrome. They concluded that high sensitivity CRP levels is a significant predictor of standard markers for myocardial damage and it may be a useful prognostic marker in acute coronary syndromes.

Table 10: studies on sensitivity and specificity of hs-crp.

\begin{tabular}{|l|l|l|l|}
\hline Authors & Year & Sensitivity & Specificity \\
\hline Reza Madadi et al $^{99}$ & 2013 & $77 \%$ & $77 \%$ \\
\hline Ayman Al-Salehet et al $^{100}$ & 2014 & $95 \%$ & $95 \%$ \\
\hline In present study & --- & $72.7 \%$ & $62.2 \%$ \\
\hline
\end{tabular}

These data suggest that increase in levels of hs-CRP is well correlated with Troponin I, CK-MB. hs-CRP is a good marker of myocardial damage but it is not as much as sensitive as Troponin $I$ in diagnosis of acute myocardial infarction.

Changes in LDH in patients complaining of chest pain:-

In present study, it was found that out of 55 patients who actually diagnosed to have acute myocardial infarction, LDH was elevated in all 36 patients. In 19 patients in spite of having acute myocardial infarction it remained in normal range. While in 45 patients who diagnosed to have other condition than acute myocardial infarction, LDH was elevated in 18 patients and in 27 patients it remained in normal range. (table 4). 
In present study sensitivity and specificity of LDH for diagnosis of acute myocardial infarction was $65.4 \%$ and $60 \%$ respectively. When applying chi-square $\left\{\mathrm{X}_{2}\right\}$ test to compare sensitivity and specificity of LDH with Troponin I the difference was statistically highly significant. ( $\mathrm{P}$ value $<0.0001$, table 11 ).

These results are comparable with other studies:

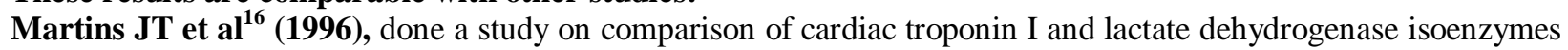
for the late diagnosis of myocardial injury. In this study, the sensitivity of cardiac troponin I (cTnI) and LD1/LD2 were compared as late markers of myocardial injury over a 5-day period in 36 patients admitted with a diagnosis of myocardial infarction to the coronary care unit. Over this period, the sensitivity of cTnI was significantly greater than that of LD1/LD2 (P < 0.05). They concluded that cardiac troponin I (cTnI) has greater sensitivity than lactate dehydrogenase isoenzymes for delayed diagnosis sof myocardial injury and is a more cost-effective test, the authors recommend it as a test of choice in this setting.

Table 11: studies on sensitivity and specificity of ldh.

\begin{tabular}{|l|c|c|c|}
\hline Authors & Year & Sensitivity & Specificity \\
\hline Fitzgerald RL et al & & $80 \%$ \\
\hline Jaffe AS et al $^{87}$ & 1996 & $67 \%$ & -- \\
\hline In present study & 1996 & $90 \%$ & $60 \%$ \\
\hline
\end{tabular}

These data suggest that $\mathrm{LDH}$ is a marker of myocardial damage but not as much sensitive and specific as Troponin I, CK-MB.

\section{Conclusion:-}

Based on present study, it is concluded that acute myocardial infarction require immediate diagnosis and intervention. In early diagnosis cardiac markers are very useful to differentiate acute myocardial infarction from other conditions.

Among the cardiac markers studied Troponin $I$ is the most specific and sensitive for diagnosis of acute myocardial infarction. As CK-MB levels become normal within 2-3 days it is helpful in diagnosis of reinfarction. hs-CRP and LDH are also the markers of myocardial damage. So for the early diagnosis and intervention of acute myocardial infarction these markers are very helpful.

\section{Bibliography:-}

1. Harrison's principles of Internal medicine $16^{\text {th }}$ edition; vol 2; chapter -288 ; ST Segment elevation myocardial inflammation; page - 1448 .

2. Ramzi S. Cotran, Vinay Kumar, Stanley Robbin's : Pathologic basis of disease. Fifth edition, prism publishers, 524-534, 1994.

3. Adam D. Timmis : Will, Serum enzymes and other proteins find a clinical application in the early diagnosis of MI. British Heart Journal 19: 309-311, Oct. 1993.

4. Hess J. W. MacDoral R.R. : Serum creatinine phosphokinase. Mitch. Medicine, 62 : 1095, 1963.

5. Antmam EM, Tenasijevic MJ, Thompson B. et al (October 1966). "Cardiac specific troponin I levels to predict the risk of mortality in patients with acute coronary syndromes.” N. Engl. J. Med 335 (18) : 1342 - 9.

6. Adams JE 3rd, Bodor GS, Dávila-Román VG, Delmez JA, Apple FS, Ladenson JH. Cardiac troponin I.A marker with high specificity for cardiac injury.Circulation. 1993;88(1):101-6.

7. Adams JE $3^{\text {rd }}$, Schechtman KB, Landt Y, Ladenson JH, Jaffe AS. Comparable detection of acute myocardial infarction by creatine kinase MB isoenzyme and cardiac troponin I. Clin Chem. 1994;40(7):1291-5.

8. Brogan GX Jr, Hollander JE, McCuskey CF, Thode HC Jr, Snow J, Sama A, Bock JL. A study on evaluation of a new assay for cardiac Troponin I vsCreatine kinase-MB for the diagnosis of acute myocardial infarction. AcadEmerg Med. 1997 Jan;4(1):6-12.

9. ShazibPervaiz, Philip Anderson, Thomas P. Lohmann, Charlotte J. Lawson, Yue-Jin Feng, Dave Waskiewicz. Comparative Analysis of Cardiac Troponin I and Creatine Kinase-MB as Markers of Acute Myocardial Infarction.Clin.Cardiol. 1997;20:269-271.

10. DurdiQujeq. Rapid Diagnosis of Acute Myocardial Infarction.Iran. Biomed. J. 1999;3(1):59-62 
11. Ross G, Bever FN, Uddin Z, Hockman EM. Troponin I sensitivity and specificity for the diagnosis of acute myocardial infarction.J Am Osteopath Assoc. 2000 Jan;100(1):29-32.

12. Kiran R Bagale1, Avinash S. Ingle. Role of CK-MB and Troponin-I in Diagnosing Non-ST-Elevation Myocardial Infarction.International Journal of Science and Research. 2014;3(8). Paper ID: 02015352.

13. Janice Zimmerman, Robert Fromm, Denise Meyer, Ann Boudreaux, Chuan-Chuan C. Wun, Richard Smalling, Barrry Davis, Gabriel Habib, Robert Roberts. Diagnostic marker cooperative study for the diagnosis of myocardial infarction.Circulation. 1999;99: 1671-1677.

14. Bock JL, Brogan GX Jr, McCuskey CF, Thode HC Jr, Hollander JE, Gunther T. Evaluation of CK-MB isoform analysis for early diagnosis of myocardial infarction.J Emerg Med. 1999 Jan-Feb;17(1):75-9.

15. Zohair Al Aseri, Syed ShahidHabib, Abdullah SalehAlhomida, Haseeb Ahmad Khan. Relationship of high sensitivity C-reactive protein with cardiac biomarkers in patients presenting with acute coronary syndrome.JCPSP. 2014;24(6):387-91.

16. Martins JT, Li DJ, Baskin LB, Jialal I, KefferJH.Comparison of cardiac troponin I and lactate dehydrogenase isoenzymes for the late diagnosis of myocardial injury. Am J ClinPathol. 1996;106(6):705-8 